\title{
Computer Graphics in Germany
}

\section{Dietmar Saupe \\ Institut für Informatik Universität Leipzig}

\section{Marc Alexa \\ GRIS}

\section{Technische Universität Darmstadt}

\section{Introduction}

In Germany we are witnessing for some 20 years an increasing interest in computer graphics, animation, art, visualization and hardware. Initially in the ' 80 s, the growth of computer graphics groups at German universities was slow. But then, sometime in the early ' 90 s, a critical point-of-no-return was reached. Since then it seems as if every computer science department has been striving to install a graphics group, if it did not already have one. In addition, centers of research and development in computer graphics are operating within institutions that are funded publicly or by industry.

A prominent example of the rapid development and joint activities of universities and research centers are the institutions located in Darmstadt. In 1975 Prof. José Encarnação founded the Interactive Graphics System Group. To involve industrial partners in computer graphics research he established the Computer Graphics Center (ZGDV) in 1984. Three years later, the Fraunhofer Society opened its doors with a work group, which led to the Fraunhofer Institute for Computer Graphics. Since then, other institutions in Germany and abroad have joined what is now called the INI-GraphicsNet, one of the world's largest research institutes in computer graphics (300 full time employees, 450 research assistants, DM 72 million budget in 2000).

The prestigious Max-Planck-Gesellschaft founded a new research group on computer graphics within the Max-Planck-Institute of Computer Science on the campus of the University of the Saarland, headed by Prof. HansPeter Seidel. Also the Konrad-Zuse-Zentrum (ZIB) in Berlin hosts a strong visualization group. Two years ago the German Research Center for Artificial Intelligence (DFKI) found- ed a research institute for visualization and simulation headed by Prof. Hans Hagen. Other public institutions with computer graphics research activities include the German National Research Center for Information Technology (GMD), housing research groups with a focus on virtual environments and digital media productions.

The German Research Foundation (DFG) recognizes the need and potential of research in computer graphics. For example, besides financing numerous individual grants, the DFG sponsors a special interdisciplinary graduate study program on 3D image analysis and synthesis at the University of Erlangen-Nuremburg. This includes funding for a yearly international workshop on Vision, Modeling and Visualization (VMV), which this year takes place in Stuttgart.

Besides the strong research development in graphics there are also interesting new avenues in graphics education. Typically, the courses on computer graphics are integrated in the standard computer science diploma curriculum. However, at the Otto-von-Guericke University in Magdeburg a new curriculum called Computational Visualistics was installed in 1996. Students in this course of studies are prepared for all occupations where a systematic creation, processing and handling of digital images is crucial. Besides technical and scientific courses students also attend courses in social sciences and humanities focusing on human interaction with imagery in social contexts. This new course of studies received an overwhelming response.

The growth of interest in graphics is also reflected in the development of the special interest group FA4.I for computer graphics in the German Association for Informatics (Gesellschaft für Informatik). Founded in 1983 by José Encarnação, it brings together about 40 representatives from universities, colleges and industry, that coordinate activities from five subgroups specializing in foundations and systems, imaging and visualization, graphical user interfaces, simulation and animation, and geometric modeling. Numerous conferences and workshops are run each year by these subgroups. Of course, the FA4.I supports Eurographics, and in particular next year's Eurographics conference, which is scheduled to take place in Saarbrücken in September 2002.

In the following section we present a small cross section of computer graphics research activities from all over the country, arranged alphabetically by city. Clearly, it would be an impossible task to include all that is relevant. Please regard our selection as an appetizer and browse for additional information from these and other places. The world wide web easily provides the required resources. We invite you to visit and follow a collection of links that also include groups with interesting research which could not be mentioned in this short column. This list is maintained by the graphics group at the University of Dortmund, see Is7-www.informatik.uni-dortmund.de/cgotn/.

In the last section we describe a few largescale graphics development projects that are supported by several German Ministries and that involve academic and industrial partners.

\section{Research at Universities and Institutes}

Complex 3D-Models at Aachen

Research projects in Aachen (Prof. Leif Kobbelt) are concerned with the generation, reconstruction, modification, storage and transmission of highly complex 3D models. Such models can be represented explicitly by mathematical descriptions (e.g. splines in CAD/CAM), procedural descriptions (subdivision surfaces) or plain polygonal meshes. Implicit representations in terms of volume data or unstructured point clouds are also common. The challenge is to design scalable algorithms that enable the efficient handling of large data sets. Hierarchical approaches are the appropriate techniques in this context since they allow the user to adapt the model complexity to the specific quality requirements and to the available hardware resources. Themes of the research are triangulation of point clouds, mesh decimation, subdivision surfaces, fairing of polygonal meshes, multiresolution modeling and data 


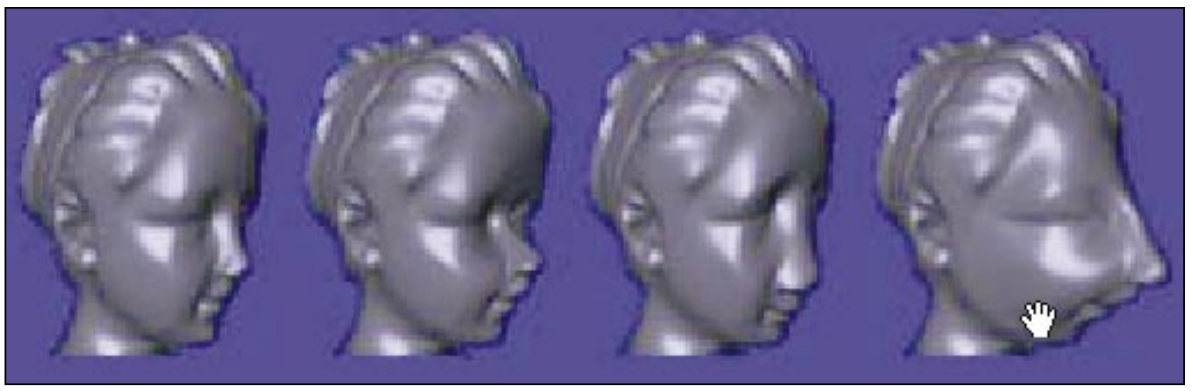

Figure I: Multiresolution modeling.

structures for efficient handling of polygonal meshes.

Web: www.informatik.rwth-aachen. de/l8.

Scientific Visualization in Berlin

Department of Scientific Visualization at the Konrad-Zuse-Zentrum in Berlin (ZIB) develops and implements new visualization techniques - utilizing powerful computer graphical and numerical algorithms (Contact: Hans-Christian Hege). Special emphasis is on innovative methods for visualization of scalar, vector and tensor fields, as well as image analysis and geometry reconstruction. Currently the focus is on application domains in medicine, biology, biochemistry and physics. For these highly interactive software systems are developed that integrate segmentation of image data, surface and volume grid generation, numerical simulation and visualization. All these virtual laboratories are built with Amira, a software framework for image segmentation, 3D geometry reconstruction and data visualization.

The department has significantly contributed to the success of the line integral convolution method for vector field visualization [13], for example, by extending the method to arbitrary surfaces; see Figure 2. They also introduced the illuminated field

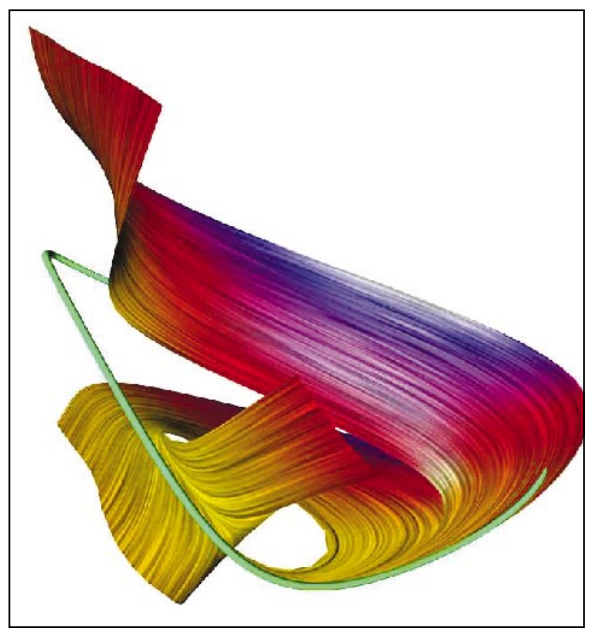

Figure 2: Line integral convolution on a surface. lines [14] for 3D vector field visualization, which were the starting point for further hardware-accelerated shading and lighting models.

Web: www.zib.de/visual.

\section{Computer Graphics and Digital Libraries} in Braunschweig

The Institute for Computer Graphics was founded in May 1998 and is directed by Prof. Dieter W. Fellner. The main research areas of the Institute currently are graphical aspects of Internet-based multimedia information systems, software architectures for integrated modeling and rendering, and efficient algorithms for interactive rendering and photorealistic visualization.

Graphical Aspects of Internet-based Multimedia Information Systems: The Institute is currently leading the strategic research initiative on 'Digital Libraries' funded by the German Research Foundation (DFG) over a period of six years consisting of a group of roughly 50 researchers working on integrating various media types - like 3D graphics, audio, video, animations - as first class citizens into digital documents and libraries.

Data Structures and Software Architectures in Computer Graphics: The Institute's work over the past years focused on increasing the general accessibility of computer graphics by algorithmic modularization supported by appropriate software architectures. Specific topics were customization of editing systems, consistent user interface management systems, configurable visualization systems and, finally, affordable (semi-) immersive projection environments. In addition to the benefit of modular tools, the major potential of this approach is the consistent modeling of complex scenes. In particular, the Institute is working on the elimination of bottlenecks between modeling and visualization. Results of this work are a new model driven and energy driven approach for discontinuity meshing in radiosity algorithms (see Figure 3 ) or a near-interactive simulation tool for the propagation of radio waves in urban environments relevant to cellular phone providers.

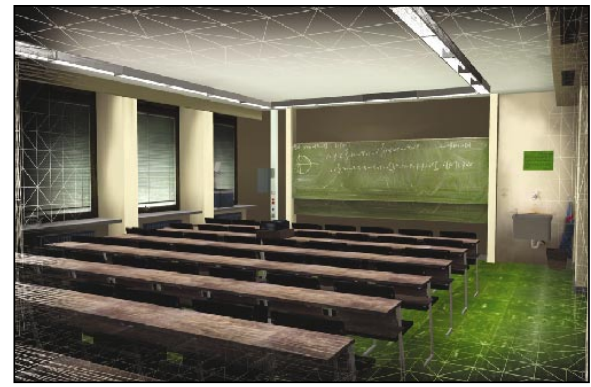

Figure 3: A new approach for discontinuity meshing in radiosity.

Information Visualization at the University of Constance

At the Computer and Information Science Department of the University of Constance, computer graphics and visualization research focuses on information visualization techniques for the visual exploration of very large data sets (Contact: Prof. Daniel Keim). The research program concentrates on visual data mining, graph visualization and human computer interaction techniques. The research is driven by collaborations with leading international research labs and commercial partners including HP Labs, AT\&T Labs and various application partners. An acquisition of an ultra-high resolution multi-projector powerwall display is currently under way.

Web: www.inf.uni-konstanz.de.

\section{Computer Graphics in Darmstadt}

Darmstadt is the center of the INI-GraphicsNet, an international alliance of institutions in computer graphics. Located in Darmstadt are the Interactive Graphics System Group (GRIS) as part of the Department of Computer Science at the Technische Universität Darmstadt, the Computer Graphics Center (ZGDV) and the Fraunhofer Institute for Computer Graphics (IGD). Further, several spin-off companies and so-called forums all working in the area of computer graphics are situated in Darmstadt.

\section{Web: www.inigraphics.net.}

The main tasks of GRIS are to teach and to conduct basic research. Current research interests span conversational user interfaces, tele-teaching and -learning, information visualization, morphing (see Figure 3 and [I]), modeling and more.

Web: www.gris.informatik.tu-darmstadt.de.

The ZGDV is a non-profit organization for the advancement of computer graphics. Members of the organization are over 30 renowned institutions from industry, business and research. Besides the institution in Darmstadt, computer graphics centers have been established in Rostock and Coimbra, Portugal.

Web: www.zgdv.de. 

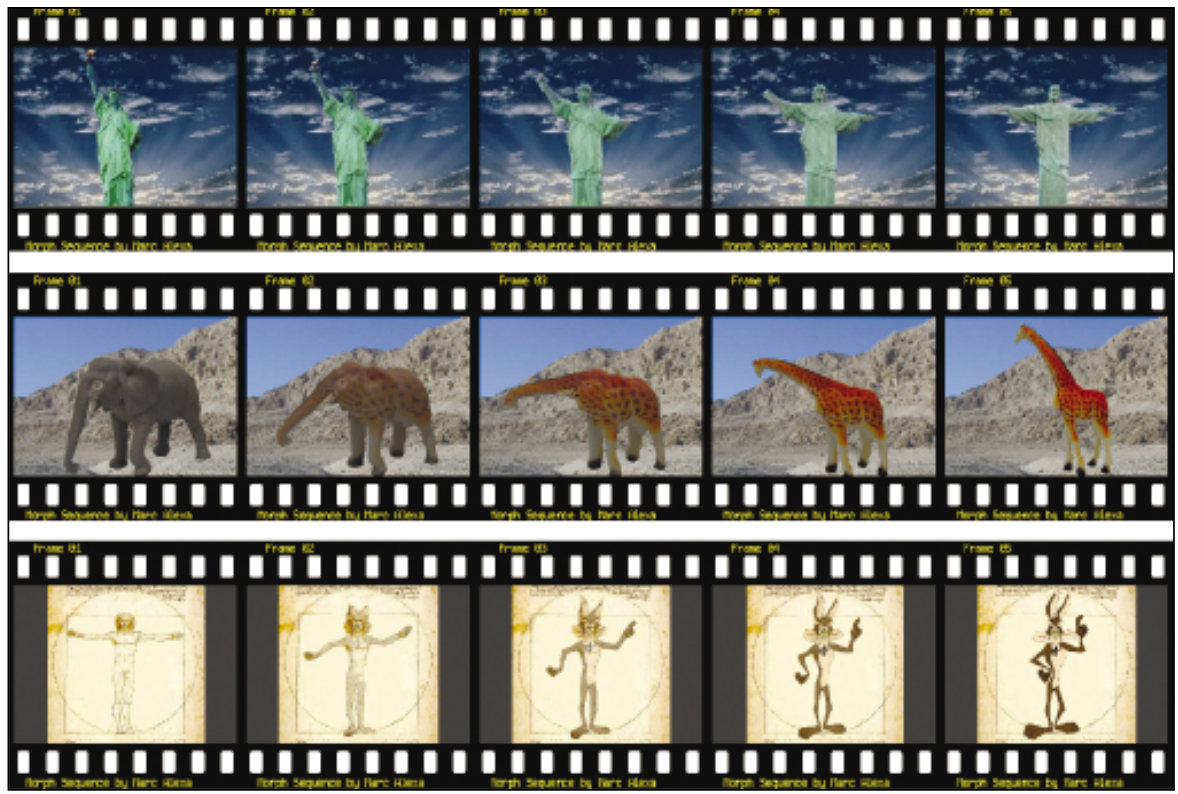

Figure 4: As-rigid-as-possible shape transformations.

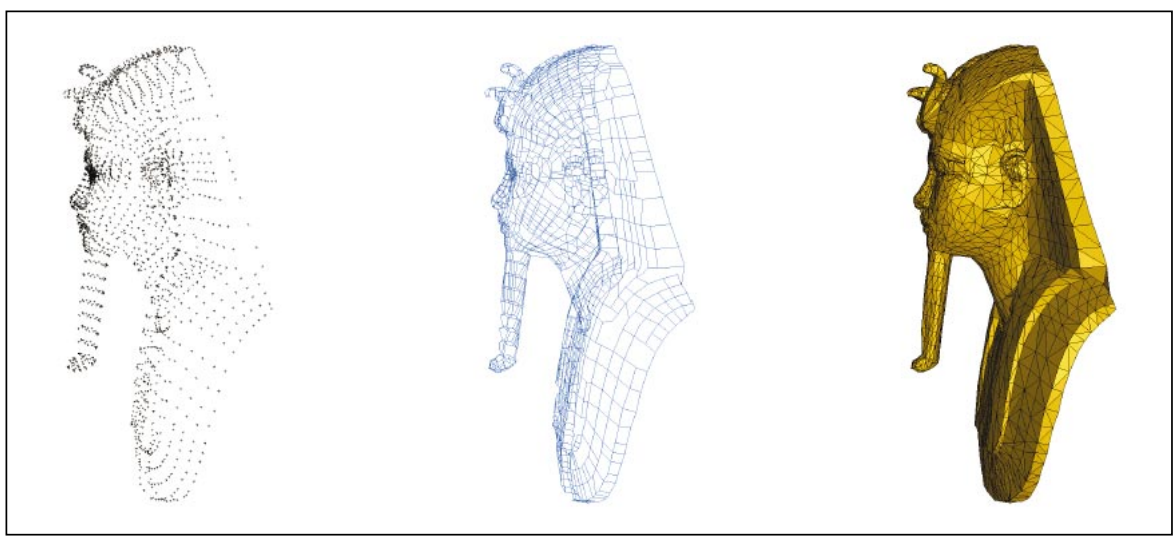

Figure 5: Reconstruction of surfaces from scattered sample points.

The spectrum of studies conducted at the IGD ranges from application-specific fundamental research, e.g., algorithms, to the creation of prototypes of applications and systems (hardware and software) as well as their adaptation to specific customer requirements. Through its research and development work, the institute helps to establish computer graphics around the world as a technology, as a tool and to influence it with its own products and methods. The R\&D projects relate directly to current problems in industry, trade, traffic and service.

Web: www.igd.fhg.de.

Several spin-off companies turn recent research results into products. The CAPCom Technology Consulting, Development and Distribution $\mathrm{GmbH}$ was the first INIGraphisNet spin-off. It helps to bring to the market those developments that have not yet been capitalized upon by specialized spin-offs. Such companies include MediaSec Technologies LLC concentrating on the field of data security in the multimedia branch, MedCom offering immersed visualization solutions. medcom-online,vrcom].de.

Algorithms and Data Structures for Graphics ization and image analysis. includes efficient manipulation of large models and immersive user interaction at a stereographic projection wall. A second system is VTM - Vision-based Tracking Module. VTM is a generic software module for human-computer interaction using computer-controlled surveillance cameras. It is based on experience with several other systems which have been developed in the past seven years.

Figure 5 shows snapshots from a surfaceoriented algorithm for reconstruction of surfaces from scattered sample points (left). In a first step, a surface graph is determined (middle). In a second step the surface graph is incrementally triangulated (right). The method is particularly suited for coarse data sets, and can be used for point-based interactive surface modeling, too. The reconstruction properties of the algorithm have been theoretically verified.

Web: Is7-www.informatik.uni-dortmund.de.

\section{Computer-Generated Plants and Landscapes} at Dresden

Synthetic plants are needed at various places where images of outdoor scenes are to be generated. Examples are special effects for entertainment or advertising as well as visual simulations in areas like architecture or landscaping. In the lab at the Technical University of Dresden (Prof. Oliver Deussen) work is performed on efficient modeling methods for realistic ecosystems, fast rendering of complex geometry, visibility culling and levelof-detail methods for plants [3]; see Figure 6 for an example. Besides realistic image generation, sketched drawings of plants is also pursued [4].

Web: www.inf.tu-dresden.de/cgm.

specializing in applications for the medical field (most notably the "InViVo"-family with the EU IST Grand Prize winner TelelnViVo) or vrcom

Web: www.[capcom,mediasec, at Dortmund

The computer graphics group at the University of Dortmund was founded in October 1992 by Prof. Heinrich Müller. Its research focuses on the development of efficient algorithms and data structures for various problems of computer graphics including geometric shape modeling, surface reconstruction (see Figure 5), surface matching, computer-aided production, rendering, visual-

Part of the research is performed in the framework of application scenarios. One system currently in the works is the GBM Grid Based Modeler. GBM is a raster-volumeoriented interactive modeling system which
Computer Aided Face Engineering in Freiburg Freiburg (Prof. Thomas Vetter) recently introduced the concept of correspondence based face space. The technique derives a general flexible face model, which allows you to synthesize novel realistic $3 D$ models of human faces [2]. The flexible model is obtained in an automated learning procedure from the example faces of a 3D face database. The procedure extracts the common structure of all faces, the prototype face, and codes individual faces through their deviation from this prototype. The model is mainly based on the linear combination of examples.

Web: graphics.informatik.unifreiburg.de/CAFE.html.

Scientific Visualization and Geometric Modeling in Kaiserslautern

Kaiserslautern hosts the computer graphics institute at the University of Kaiserslautern and the Intelligent Visualization and Simulation
The research group at the University of 


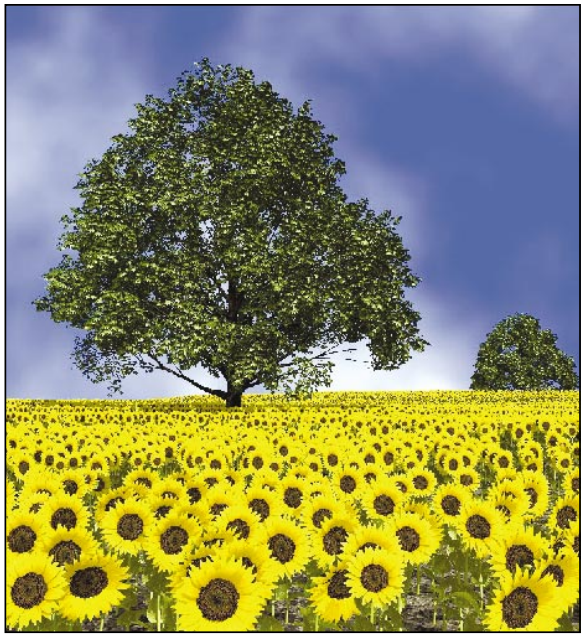

Figure 6: Modeled sunflower field.

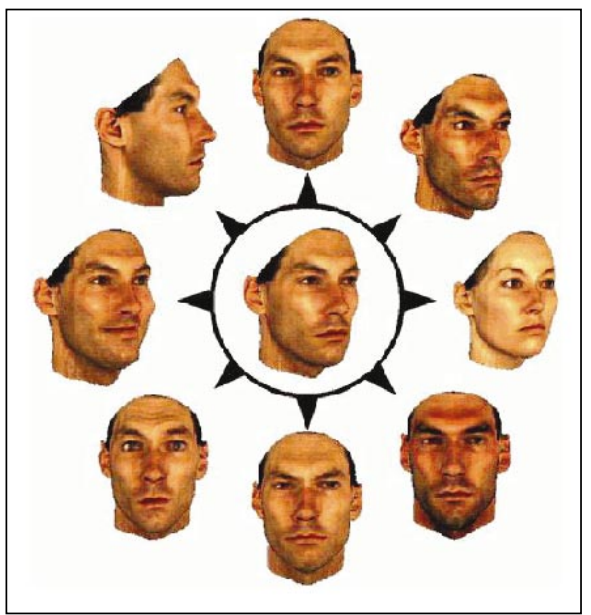

Figure 7: Face Space.

Systems (IVS) department of the German Research Center for Artificial Intelligence (DFKI), both headed by Prof. Hans Hagen.

The computer graphics institute at the University of Kaiserslautern does basic and applied research in geometric modeling and scientific visualization. The geometric modeling group focuses on variational design, physically and simulation based modeling, triangulation algorithms, scattered data methods and hierarchical modeling. It cooperates with several companies, especially in the automotive industry and has led to the foundation of two spin-off companies. The scientific visualization group concentrates on the visualization of large unstructured data sets, hierarchical visualization, web-based visualization systems, and vector and tensor field visualization. Figure 8 shows the visualization of complex chemical molecules. The group has substantially contributed to the topologybased analysis and visualization of vector and tensor field data, e.g., the detection of closed streamlines [19] or the topological visualization of time dependent fields.

Web: davinci.informatik.uni-kl.de.

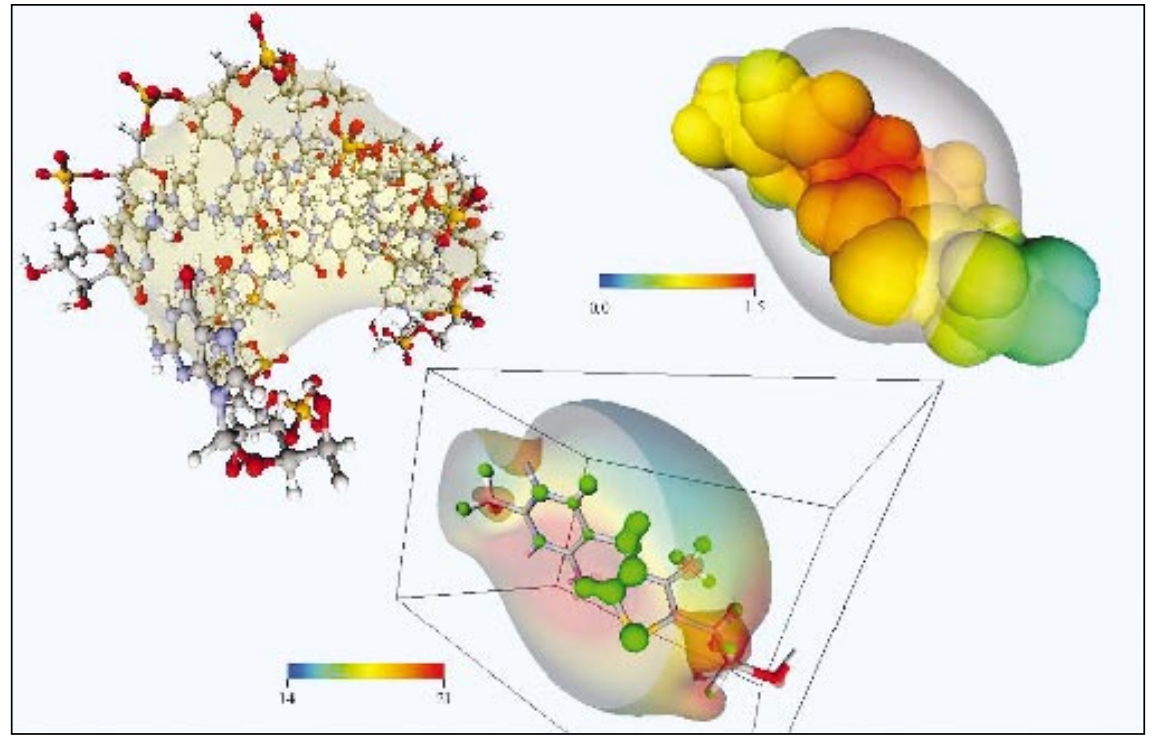

Figure 8: Visualization of complex chemical molecules.

The department for intelligent visualisation and simulation (IVS) of the German Research Center for Artificial Intelligence (DFKI) develops interactive techniques to visualize subjects and concepts both in the artificial intelligence environment and in technical simulations. A special area of interest is the elaboration of virtual reality methods, which have applications in the field of human modeling and medical information systems.

One main topic of IVS is the development of a visualization system architecture that includes an intelligent control unit capable of supervising and tuning all system components during runtime. Therefore, multi-agent technology and component-based implementation is combined [19]. In the field of medical computer science, clinical information systems and electronic patient records as well as medical image processing and visualization tasks are developed. The human modeling division is applying virtual human models in the different stages of product life cycle. Because of the ability to vary the anthropometry of the human model, a whole group of virtual humans can be used to perform product assessment without significant additional effort. At IVS, methods for an improved realistic representation of the human body are developed and analysed.

Web: www.dfki.de/ivs2/IVS_Englisch.

\section{Visual Communication of Information} at Magdeburg

The Graphics and Interactive Systems Laboratory at the University of Magdeburg was founded in October 1993 and is directed by Prof. Thomas Strothotte. The long-term goal of the activities is to make communication through computer-generated images into a flexible medium of expression. This requires methods and tools for rendering expressive computer graphics and a clarification of the relationship between images and text. Research focuses on rendering (in particular non-photorealistic rendering), visual communication of information (in particular imagetext coherence) and extraordinary interfaces (tactile graphics and synthetic holography). In order to investigate the relationship between image and language, it aspires to generate images which are as close to language as possible, namely images whose significance can be verbalized by the users in an easy, correct and adequate way.

Web: isgwww.cs.uni-magdeburg.de/ graphik.

Modeling and Visualization at Rostock

Computer graphics has a long tradition at the University of Rostock. It began in 1969 when a small group of scientists and students started to work on problems of graphical data processing. Today's Institute of Computer Graphics is headed by Prof. Dietmar Jackél. The research work focuses on the visualization of information and large data sets, on adaptive image generation, transfer and display as well as on physically-based modeling and animation. We name three examples. A scalable framework for information visualization has been developed to explore large heterogeneous information spaces at arbitrary levels of detail including a suitable preprocessing of information quantities and the combination of different graphical interfaces.

Second, a new demand-driven image transmission scheme has been developed for mobile environments with low transmission bandwidth. This schema works with regions of interest and levels of detail and is connected with a new focus and context technique to display the image on small screens. Another 


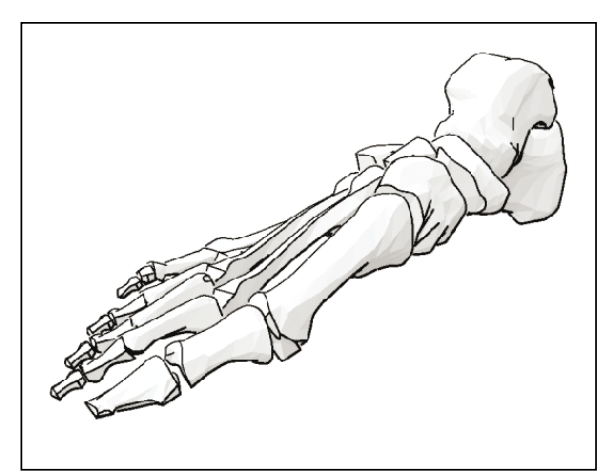

Figure 9: Human foot: Combination of line drawing graphics with flat shading.

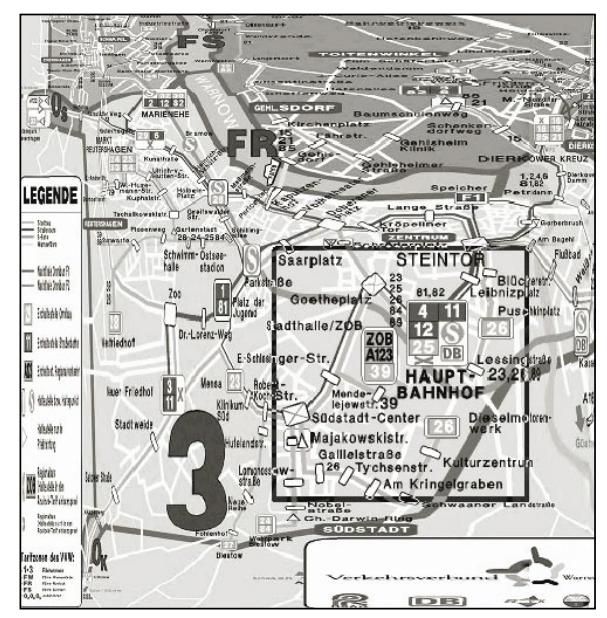

Figure 10: The focus and context technique for mobile environments saves screen space and transmission bandwidth.

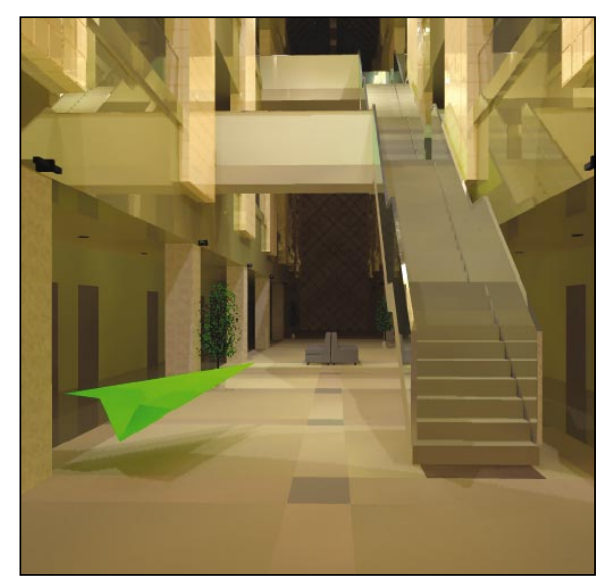

Figure II: Example frame from the ATRIUM sequence with temporal processing and spatial filtering (SIGGRAPH 200I).

framework allows the physical-based specification of realistic object movement in a very simple way by the user. It is based on the Lagrange factor method and offers a wide variety of movement control and interaction techniques.

Web: wwwicg.informatik.uni-rostock.de.

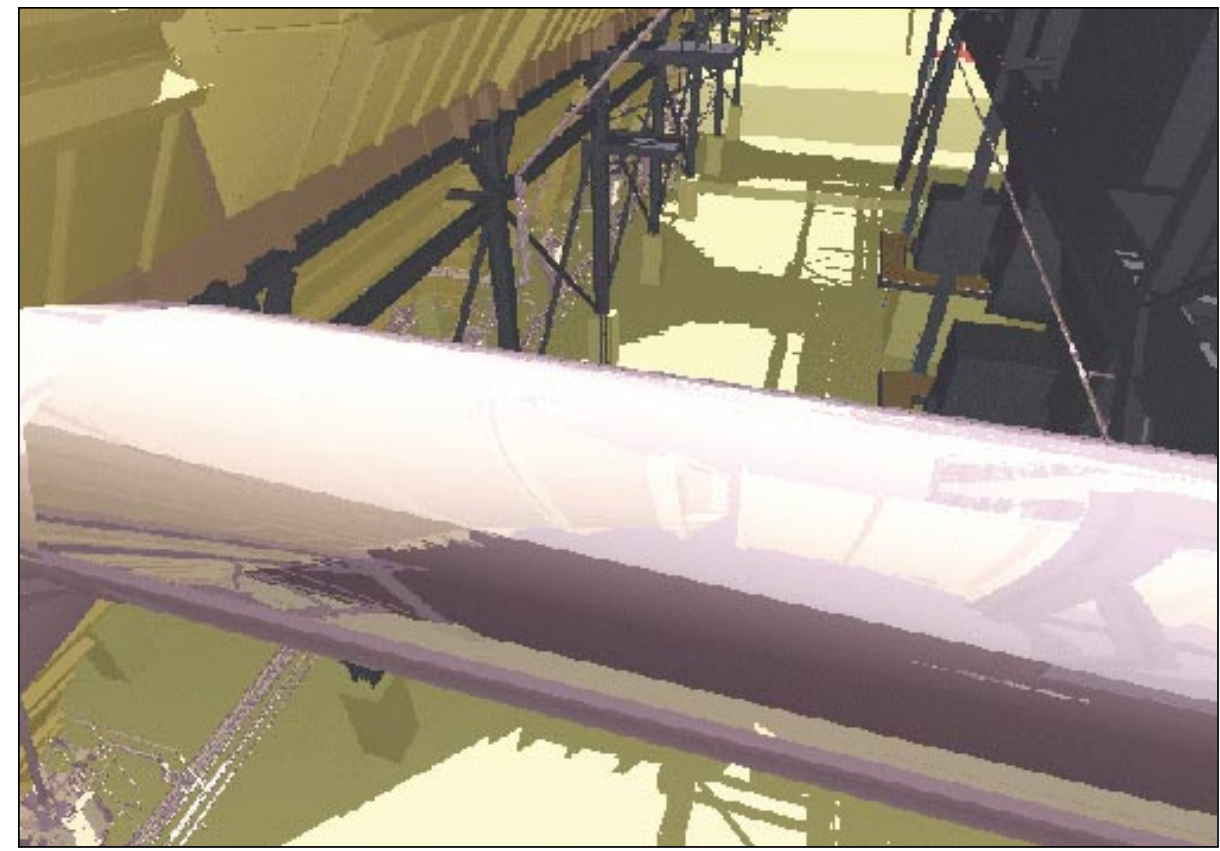

Figure 12: Detail view from an interactive walkthrough with shadows and reflections of the entire UNC power plant model, which consists of I 2.5 million triangles.

Research at the Max-Planck-Institute for Computer Science in Saarbrücken

The Max-Planck-Institut for Computer Science (MPI) is one of the leading research institutes in the world for cutting-edge research in computer science and information technology. The MPI computer graphics group was founded in 1999 with the appointment of Prof. H.-P. Seidel to the position of scientific director. The graphics group currently consists of three faculty, six postdocs and 17 researchers.

At its last meeting the International Scientific Advisory Board of the institute has rated MPI as the best institute of its kind in the world. MPI is knowledge oriented and open to applied research in computer science, and it is part of the German infrastructure for cutting-edge research in computer science that includes the Max-Planck-Institut (MPI), the Dagstuhl International Conference and Research Center for Computer Science (IBFI), the German Research Center for Artificial Intelligence (DFKI) and the large Computer Science Department at the Universität des Saarlands (all conveniently located within direct reach of each other). In a recent evaluation of all German computer science deparments by external faculty members, conducted by the Center for University Development (HE), researchers at MPI and at the computer science department at the Universität des Saarlands have been ranked number one nationwide.

MPI has been designated a EU Marie Curie training site and the group participates in various research projects on a national and international level, funded by the German
National Research Foundation (DFG), the European Community (EU), the Federal Goverment (BMBF), NATO and the GermanIsreal Foundation (GIF), among others. The group also collaborates closely with several industrial partners, such as BMW, Mercedes, Motorola and Siemens.

Research within the group currently focuses on computer graphics, geometric modeling, freeform curves and surfaces, surface reconstruction, efficient polygonal meshes, mesh reduction, multiresolution modeling, image synthesis, global illumination computations, image-based and hardwareaccelerated rendering, visualization of complex medical and engineering data, 3D image analysis and synthesis and foundations of virtual reality.

The scope of MPI is truly international, and the group has kept a steady presence at all major computer graphics events, such as SIGGRAPH, Eurographics, etc. Three typical publications in this regard deal with hardware accelerated rendering [7], feature sensitive data acquisition [8] and perception guided global illumination [10].

EUROGRAPHICS'2002, the annual conference of the European Association for Computer Graphics, in 2002 will be hosted by MPI and the Universität des Saarlands in Saarbrücken.

\section{Web: www.mpi-sb.mpg.de/units/ag4.}

Interactive Ray-Tracing in Saarbrücken

The computer graphics lab at the Universität des Saarlandes in Saarbrücken was founded by Prof. Philipp Slusallek in October 1999. The group currently consists of seven researchers 


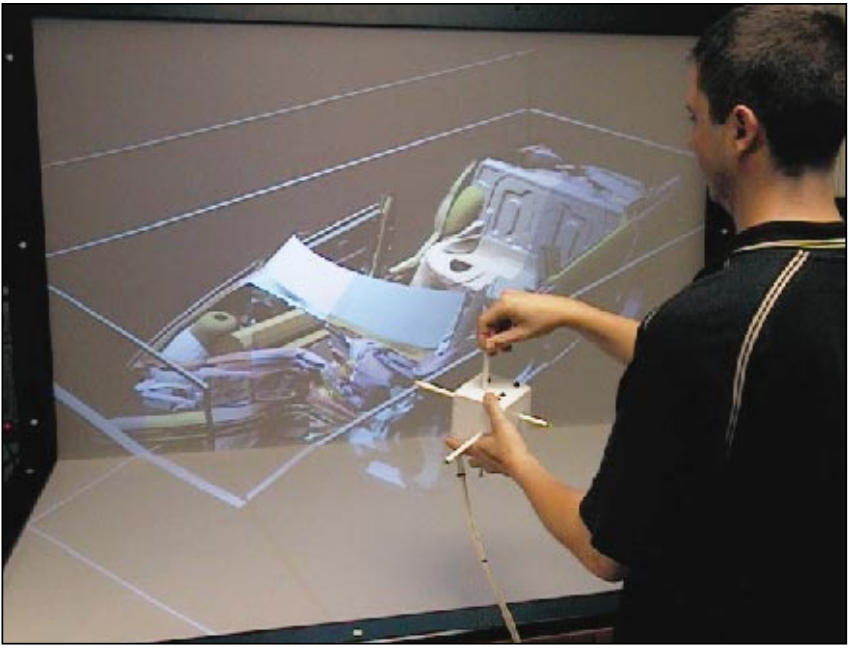

Figure 13: The Cubic Mouse for intuitive control in VR applications.

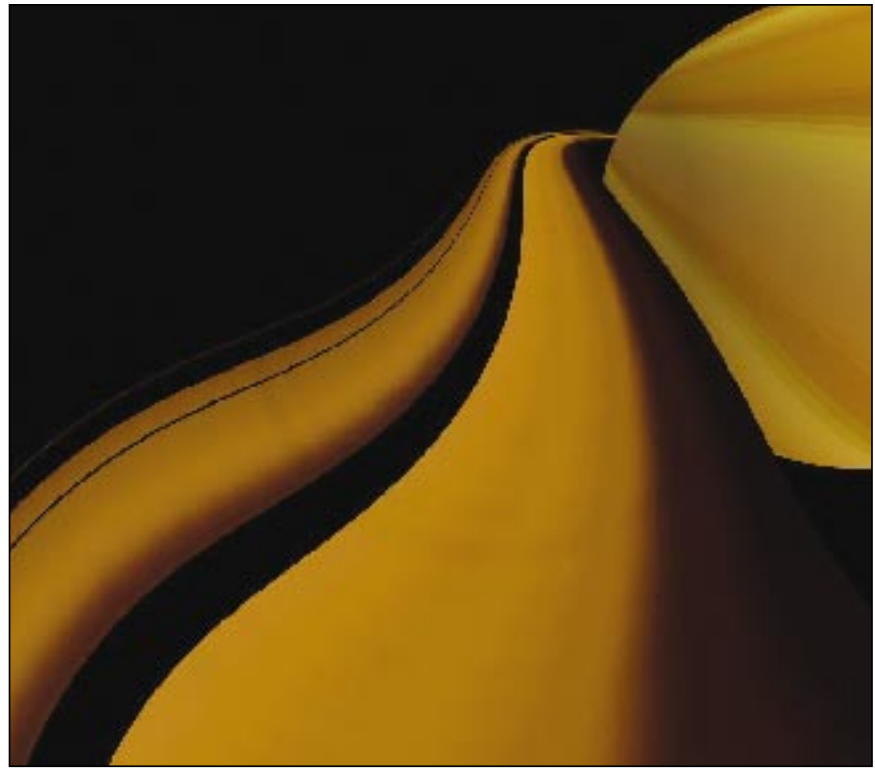

Figure 14: Snapshot from a flyby of Saturn at 99 percent speed of light. working mainly in the areas of real-time raytracing, realtime global illumination for mixed reality and virtual studio applications, as well as distributed multimedia middleware.

A key research topic is the use of ray-tracing for interactive 3D graphics. The group has built the world's fastest ray-tracer through a mixture of new algorithms that better expose coherence in the ray-tracing algorithm, making better use of caches and SIMD extensions of current CPUs, and distributing scene data and computations in a cluster of workstations. Even on a single machine this interactive system improves performance over other software ray-tracers by a factor of about 15 and beats even the best graphics hardware for complex environments [16].

In another project sponsored by Motorola, the group is designing a distributed multimedia infrastructure. This system will allow applications to take advantage of multimedia devices distributed throughout an Intranet or even the Internet as if they were connected to the local computer. This multimedia infrastructure is also the basis for the ongoing research on global illumination for virtual studio and similar setups. Here the challenge is to measure, recompute and apply illumination in real time in both the real and virtual scenes in order to keep the lighting in the two worlds consistent.

Web: graphics.cs.uni-sb.de.

\section{Virtual Environments at the GMD}

in Sankt Augustin

The Virtual Environments (VE) Competence Center has its roots in the VMSD Research Division which was established by Wolfgang Krüger in 1991 and is headed by Dr. Martin Göbel. Research and development of this group is focused in the areas of systems development, input technologies and interaction techniques, display technology, visualization and sonification techniques, distributed virtual environments and art and storytelling installations.

GMD's developments are based on their object-oriented virtual environment framework Avango. Avango is implemented on top of SGl's Performer toolkit and OpenGL. It is available for IRIX and Linux systems and supports all VR-typical input and output devices. Avango augments the Performer scene graph with a field-based interface and adds a scripting layer for rapid application development. Avango also provides distribution support through scene graph replication and a field based synchronization mechanism.

The Responsive Workbench [9], an early development of GMD's virtual environment group, has become a standard display for virtual environment applications. In 1997, the twosided, L-shaped Responsive Workbench was developed in cooperation with the Germanybased TAN projection technology company. This display extends the range of workbench applications considerably by providing a larger workspace while keeping the table-top metaphor. Recently, they have developed a conic, 230 degree display in close cooperation with Barco. The display has a diameter of 6.5 meters, a height of 2.8 meters, and it is driven by four projectors. It is tilted by approximately five degrees to avoid parallel display surfaces, which is a requirement for good acoustical properties. Another focus of GMD's work is the development of acoustic displays.

GMD's Cubic Mouse is a novel input device for intuitive control of VR applications, which puts the three-dimensional coordinate system into the user's hand.This device replaced gloves and wands in most of their visualization applications. A free-hand sketching tool is currently under development to support conceptual surface based modeling in virtual environments.

For many years engineering visualization in virtual environments has been explored in cooperation with partners from the automotive and the oil and gas industry. Digital storytelling installations using GMD's CAVE-like fully immersive environment CyberStage were developed in cooperation with RMH and Vertigo Systems, two spin offs from the virtual environments group. This joint work has resulted in numerous CyberStage productions for different industrial partners. Art installations with a focus on combined visual and acoustic experiences have been an inspiring complement to the technical work over the past years.

\section{Web: imk.gmd.de.}

\section{Scientific Visualization in Stuttgart}

At the University of Stuttgart the Visualization and Interactive Systems group (VIS) headed by Prof. Thomas Ertl conducts research in scientific visualization, computer graphics and human computer interaction, often in collaboration with industrial partners such as BMW and Siemens. Some of the manifold topics are interactive volume rendering via $3 D$ textures [18] and novel PC graphics hardware, volume rendering by cell projection and for non-convex tetrahedral meshes, hardware accelerated registration, segmentation, visualization of medical data sets, interactive remote and webbased visualization, flow visualization on hierarchical grids, visualization of crash-simulations, terrain rendering, visualization and steering of chemistry and physics computations, benchmarking of OpenGL and extensions.

Einstein's theory of special relativity is widely regarded as a difficult and hardly comprehensible theory. One important reason for this is that the properties of space, time and light in relativistic physics are totally different from those in classical Newtonian physics. Computer simulations are the only means of visually exploring the realm of special relativity. They 


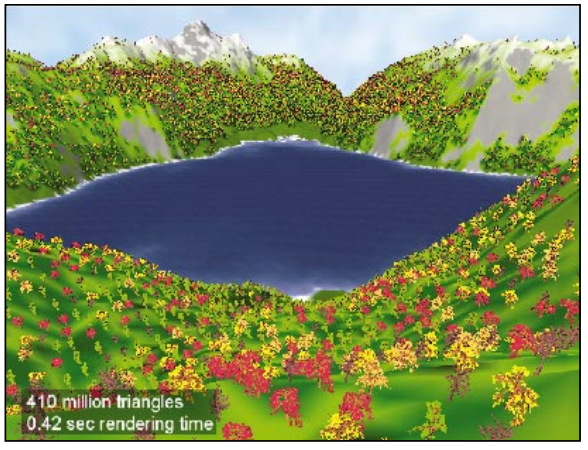

Figure 15: Millions of triangles rendered in a fraction of a second.

enable us to experience special relativistic phenomena such as Lorentz contraction, time dilation, aberration and finite speed of light. At Stuttgart several novel rendering techniques for special and general relativity have been developed; see e.g., [17] and Figure 14.

Web: wwwvis.informatik.uni-stuttgart. deleng.

\section{Computer Graphics at Tübingen}

The chair for Computer Graphics at University of Tübingen (WSI/GRIS) has been founded in 1986 by Prof.Wolfgang Straßer.Today 20 fulltime researchers work at WSI/GRIS. Highlights of graphics research at WSI/GRIS include antialising and high performance graphics hardware resulting in the first $\mathrm{PCl}$-based real-time volume renderer Vizard, anisotropic texture filtering (footprint-assembly), volume rendering hardware, textile modeling and simulation, multiresolution modeling, mesh-compression, the randomized z-buffer, multimedia-system for graphics education, all of which has been published in five SIGGRAPH conferences papers (see, e.g., $[6,15]$ ) and numerous journals. In 1986, Straßer started the successful series of Graphics Hardware Workshops.

A main research topic of WSI/GRIS is output sensitive algorithms for rendering highly complex scenes. One possibility is data reduction by view frustum and occlusion culling. GRIS has contributed to occlusion culling by algorithms that extend the OpenGl pipeline at the pixel creation level avoiding rasterization of occluded object parts. Another approach is multiresolution modeling where the level of detail, depending on the point of view, is calculated in real time. An important option is data compression for which GRIS has contributed the cutborder mesh compression machine. Another approach is the randomized z-buffer, which reduces the amount of data based on the resolution of the output medium using point sampling techniques; see Figure 15. In the area of animation, the long-term research in physically-based modeling contributes to a national industrial project developing an ecommerce system for virtual clothing.

Web: www.gris.uni-tuebingen.de.
Large-Scale Graphics Development Projects

To foster interaction of research institutes and the industry the federal goverment supports large-scale projects through its ministries of Education and Research (BMBF) and Economy (BMWi). Here, we illustrate this with a few of the many interesting projects.

\section{EMBASSI}

EMBASSI is a joint research project of 19 partners from industry and academia. Its focus is the development of new paradigms and architectures for the intuitive interaction with technical infrastructures of everyday life, such as home entertainment and control, public terminals, and car infotainment systems.

EMBASSI aims at enhancing the interaction with these infrastructures by providing intelligent assistance, multimodal interaction and anthropomorphic user interfaces within a unified framework. Technical development in EMBASSI is based on a user centered approach, with accompanying psychological and ergonomics research.

A primary objective of the project is to establish an "EMBASSI layer" that extends recently developed networking standards like $\mathrm{HAVi}$, UPnP or Konnex towards user-centered, goal-based interaction by merging experiences from knowledge based $\mathrm{Al}$ systems with those coming from device oriented command and control architectures. This layer will enable the unification of interaction paradigms and will assist the user by enabling "natural" or "intuitive" commands instead of forcing the user to think in terms of device functions.

\section{Web: www.embassi.de.}

\section{ARVIKA}

The project ARVIKA aims to research and implement Augmented-Reality technologies (AR) which will support development, production and servicing with relation to complex technical products in a user-oriented and application driven manner. Through the visual superimposition of real objects on computergenerated virtual objects, Augmented Reality technology makes it possible to act appropriately in real, in the sense of this extended reality, working situations.

The project ideas are applied in such areas as automobile and airplane construction and in machine and plant construction. These are areas of extraordinary importance for German industry. The prospects offered by this technology are of particular benefit to mid-sized companies which, through improved diagnosis and servicing capabilities, can act and react more flexibly and more efficiently and so improve their ability to compete globally.

\section{Web: www.arvika.de.}

MAP

Working environments, especially computersupported workplaces, have changed dramati- cally over time. In times of mainframe computers, the machines were operated by experts. From the ' 80 s and ' 90 s until today, computers have mainly been used by single users. In the future, the computer will not be "used" anymore but the user - who will increasingly function as an actor - will delegate tasks. The computer will be more like an assistant.

This new role of the computer is the focus of the project MAP. Together with new working structures which lead more and more to mobile work, the topics delegation, assistance as well as support of mobile devices and integrated networks will play a main role in the developments. One goal of the MAP project is the development of a kernel-system which includes the support of agent technology, new human-computer interaction mechanisms, the integration of security mechanisms and the support of mobile devices and context-aware working.

The MAP consortium consists of 10 industrial partners, including six SMEs and six research partners.

Web: www.map2I.de.

Open SG

After the Fahrenheit cooperation between Microsoft and SGI broke apart in the summer of 1999 the VR community faced a large void. To fill this void an initiative was started in the department for Visualization and Virtual Reality at Fraunhofer-IGD to write a new scenegraph, which would be able to fulfill the present and future requirements for a scenegraph in VR applications. These include easy to use and efficient multi-process and multi-processor support, simple extendibility to cope with the rapidly changing requirements for a VR system, as well as being able to adapt to the changing graphics hardware environment.

To guarantee safety for the users, it was decided to organize the project as an Open Source initiative, i.e., everybody interested in the system can freely download the source to use it, extend it and to develop it. To streamline the development direction to the user's needs and to build a financial base that supports continuous and stable development the OpenSG Forum was founded in January 2000. The Forum is a cooperation of companies and organizations that support and direct the OpenSG initiative, with about 20 members from industry, research organisations and academia.

To support the use of OpenSG in the academic area and to get new research results integrated into OpenSG, the OpenSG PLUS project was started. The project unites a large part of the German research community in the area of computer graphics and is roughly organized in four areas:

- Low-level: base support services.

- Large Scene Support: methods to handle and render large scenes. 
- High-Level Primitives: methods to support non-polygonal primitives like NURBS surfaces, subdivision surfaces and volumes.

- High-Level Shading: methods to support complex reflection models like BRDFs.

The results will be presented in the context of an annual OpenSG workshop, together with other works and news in the context of OpenSG.

Web: www.opensg.org.

\section{References}

I. Alexa, M., D. Cohen-Or, D. Levin. As-RigidAs-Possible Shape Interpolation, ACM SIGGRAPH 2000 Conference Proceedings, PP.I57-I64.

2. Blanz, V., T. Vetter. A Morphable Model for the Synthesis of 3D Faces, ACM SIGGRAPH 99 Conference Proceedings, pp. I87-I 94.

3. Deussen, O., P. Hanrahan, M. Pharr, B. Lintermann, R. Mech, P. Prusinkiewicz. Realistic Modeling and Rendering of Plant Ecosystems, SIGGRAPH 98 Conference Proceedings, pp. 275-286.

4. Deussen, O., T. Strothotte. Computer-Generated Pen-and-Ink Illustration of Trees, SIGGRAPH 2000 Conference Proceedings, PP.I3-I8.

5. Ebert, A., M. Bender, H. Barthel, A. Divivier. Tuning a Component-based Visualization System Architecture by Agents, International Symposium on Smart Graphics, (in cooperation with ACM SIGGRAPH), IBM T.J.Watson Research Center, Hawthorne, New York, NY, USA, 200 I.

6. Gumhold, S., W. Straßer. Real Time Compression of Triangle Mesh Connectivity, SIGGRAPH 98 Conference Proceedings, 1998.

7. Heidrich, W., H.-P. Seidel. Realistic, Hardware-accelerated Shading and Lighting, SIGGRAPH 99 Conference Proceedings, 1999, PP. $171-178$.

8. Kobbelt, L., M. Botsch, U. Schwaneck, H.-P. Seidel. Feature sensitive surface extraction from volume data, SIGGRAPH 200 I Conference Proceedings, 200I, to appear.

9. Krüger, W., B. Fröhlich. The Responsive Workbench, IEEE Computer Graphics and Applications, pp. 12 - I5, May 1994.

10. Myszkowski, K., T. Taware, H. Akamine and H.-P. Seidel. Perception-Guided Illumination Solution for Animation Rendering, SIGGRAPH 200I Conference Proceedings, 200 I, to appear.

I I. Peitgen, H. -O., D. Saupe. The Science of Fractal Images, Springer-Verlag, New York, 1988.

12. Peitgen, H.-O., P. Richter. The Beauty of Fractals, Springer-Verlag, Heidelberg, 1986.

13. Stalling, D., H.-C. Hege. Fast and Resolution-Independent Line Integral Convolution, SIGGRAPH 95 Conference Proceedings, pp. 249-256.

14. Stalling, D., M. Zöckler, H.-C. Hege. Fast Display of Illuminated Field Lines, IEEE
Transactions on Visualization and Computer Graphics 3:2 (1997), pp. II 8-128.

I5. Wand, M., M. Fischer, I. Peter, F. Meyer auf der Heide, W. Straßer. The Randomized zBuffer Algorithm: Interactive Rendering of Highly Complex Scenes, SIGGRAPH 200 I Conference Proceedings, 200I, to appear.

16. Wald, I., P. Slusallek, C. Benthin, M. Wagner. Interactive Rendering with Coherent RayTracing, Computer Graphics Forum (Proc. Eurographics 200I), to appear.

17. Weiskopf, D., U. Kraus, H. Ruder. Searchlight and Doppler Effects in the Visualization of Special Relativity: A Corrected Derivation of the Transformation of Radiance, ACM Transactions on Graphics 18, 3, July 1999, pp. 278-292.

18. Westermann, R., T. Ertl. Effciently Using Graphics Hardware in Volume Rendering Applications, SIGGRAPH 98 Conference Proceedings, 1998, pp. 169-179.

19. Wischgoll, T., G. Scheuermann. Detection and Visualization of Closed Streamlines in Planar Flows, IEEE Transactions on Visualization and Computer Graphics 7:2, 200 I.

\section{About the Columnists}

\author{
Alain Chesnais \\ Alias|Wavefront \\ 17 bis rue Joseph de Maistre \\ 75018 Paris, France \\ Tel: +33-|-44-92-8|-8| \\ Fax: +33-|-44-92-8|-82 \\ Email: alain_chesnais@siggraph.org \\ Jose Encarnação \\ Fraunhofer-Institut fuer Graphische \\ Datenverarbeitung \\ Wilhelminenstrasse 7 \\ D-64283 Darmstadt \\ Deutschland \\ Tel: +49-(0)6|5|-|55-|30| \\ Fax: +49-(0)6|5|-|55-430| \\ Email: jle@igd.fhg.de
}

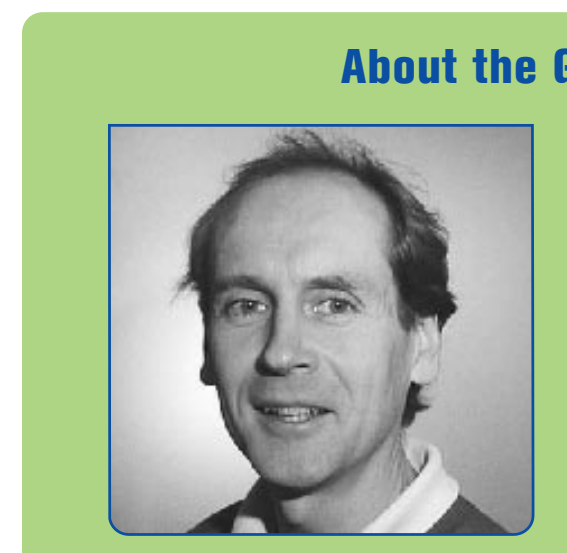

Dietmar Saupe is Chair of the Computer Graphics Special Interest Group of the German Computer Science Association (FA4.I of the Gesellschaft für Informatik). He received a diploma, a doctorate degree and the Habilitation in Mathematics at the University of Bremen. From 1985 to 1987, he was a visiting Assistant Professor of mathematics at the University of California, Santa Cruz, and subsequently Lecturer at the University of Bremen. From 1993, he was Professor of computer science at the University of Freiburg, and since 1998 at the University of Leipzig, where he is heading a research group on computer graphics and image processing. $\mathrm{He}$ has chaired and co-chaired eight SIGGRAPH courses on fractals in computer graphics from 1988 until 1996, the ACM SIGGRAPH/Eurographics Workshop on Implicit Surfaces 1998 and is Co-Chair of Eurographics 2002, Saarbrücken. The SIGGRAPH fractals courses resulted in the book The Science of Fractal Images [I I] in 1988, which served as an entry point to graphics for many young researchers at the time. Pictures of complex mathematical fractal objects were collected (see [12]) and travelled around the world for quite a number of years in an exhibition of the Goethe Institute. Saupe's current research ranges from fractal modeling of complexity in nature to efficient algorithms in scientific visualization, image, video and mesh coding and medical image processing. A focus in recent years has been fractal image compression.

Dietmar Saupe

Email: saupe@acm.org

Web: www.informatik.uni-leipzig.de/cgip

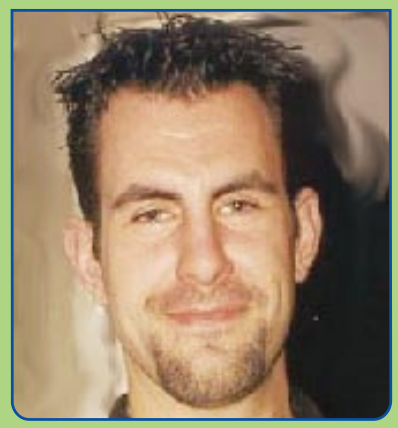

Marc Alexa leads the project group 3D Graphics Computing within GRIS, Darmstadt, and is a Ph.D. candidate in the Department of Computer Science at the Technische Universität Darmstadt. He received his M.S. degree in computer science with honors from TU Darmstadt. His research interests include shape modeling, transformation and animation as well as conversational user interfaces and information visualization.

Marc Alexa

Email:alexa@gris.informatik. tu-darmstadt.de 